\section{Acute coronary syndromes}

$J^{\mathrm{i}}$ im Christenson and colleagues, ${ }^{1}$ in their article about emergency department assessment of chest discomfort, state that chest pain evaluation units "are cost-effective relative to admitting all low-risk patients to coronary care units but have never been compared with the unstructured diagnostic approach used in most Canadian hospitals." However, more evidence for chest pain units has recently become available.

In a randomized controlled trial and economic evaluation, my colleagues and I compared a chest pain unit with routine care in the United Kingdom. ${ }^{2}$ Routine care consisted of an unstructured diagnostic approach, with hospital admission or discharge at the discretion of the physician. The chest pain unit was associated with fewer hospital admissions (37\% v. $54 \%, p<0.001)$, improved quality of life, and trends toward a lower proportion of those with acute coronary syndromes being discharged (6\% v. $14 \%$, $p=0.26)$ and lower health service costs ( $\$ 478$ v. $£ 556, p=0.25$ ). Overall, the chest pain unit appeared more effective and more cost-effective than routine care.

\section{Steve Goodacre}

Medical Care Research Unit

University of Sheffield

Sheffield, UK

\section{References}

1. Christenson J, Innes G, McKnight D, Boychuk B, Grafstein E, Thompson CR, et al. Safety and efficiency of emergency department assessment of chest discomfort. CMA7 2004;170(12):1803-7.

2. Goodacre S, Nicholl J, Dixon S, Cross E, Angelini K, Arnold J, et al. Randomised controlled trial and economic evaluation of a chest pain observation unit compared with routine care. $B M \mathcal{F}$ 2004;328:254-7.

Competing interests: None declared.

DOI:10.1503/cmaj.1041202

I $n$ a well-structured study on the contentious subject of emergency department assessment of chest discomfort, Jim Christenson and associates ${ }^{1}$ conclude that the "miss rate" for acute coronary syndromes (ACS) was $5.3 \%$ at
2 Vancouver hospitals, more than $2 \frac{1}{2}$ times that of our US counterparts.'

At first glance, this is devastating news. However, certain points might benefit from clarification. For example, of the 21 patients with ACS who were discharged from the emergency department, most had a diagnosis of chest pain not yet determined or atypical chest pain. But how many of these were discharged with empiric antianginal treatment (e.g., acetylsalicylic acid or nitroglycerin as required) and had definitive follow-up? Would such treatment of this subset of patients, if at low risk for ACS, not meet the standard of practice? ? $^{3,4}$

In the ACS patients whose condition was truly missed, what were the negative consequences of not being admitted from the emergency department? In the one case of death, what was the temporal relationship between death and the ACS diagnosis? In other words, is it likely that the outcome would have been prevented by admission on the index visit? Also, were there other adverse events in this subset of patients and, if so, is it likely that admission would have averted their occurrence?

The authors refer to the study by Pope and colleagues ${ }^{2}$ as the standard. However, I believe there are significant differences in the design of that study that would reduce the number of missed diagnoses. For example, those authors included all critically ill patients, whereas Christenson and associates, because of consent issues, did not. Pope and colleagues used only creatine kinase data in making the diagnosis, whereas Christenson and associates also used the data from the more sensitive and specific troponin assay. Pope and colleagues did not employ diagnostic data such as an outpatient stress test or angiogram results to capture more patients, but Christenson and associates did. I believe that if the design of the US study were to be used on the study population examined by Christenson and associates, the percentage of missed diagnoses would be considerably lower than $5.3 \%$.

Once it is clarified whether appropriate treatment was rendered to the "missed" group after discharge and whether admission would have prevented any adverse events, then the significance of the $5.3 \%$ figure, in isolation, can be gauged.

\section{Brian D. Steinhart}

Emergency Physician

St. Michael's Hospital

Toronto, Ont.

\section{References}

1. Christenson J, Innes G, McKnight D, Boychuk B, Grafstein E, Thompson CR, et al. Safety and efficiency of emergency department assessment of chest discomfort. CMA7 2004;170(12):1803-7.

2. Pope JH, Aufderheide TP, Ruthazer R Woolard RH, Feldman JA, Beshansky JR, et al. Missed diagnoses of acute cardiac ischemia in the emergency department. N Engl 7 Med 2000; 342:1163-70.

3. Braunwald E, Antman EM, Beasley JW, Califf RM, Cheitlin MD, Hochman JS, et al. ACC/AHA guidelines for the management of patients with unstable angina and non-ST-segment elevation myocardial infarction. A report of the American College of Cardiology/American Heart Association Task Force on Practice Guidelines (Committee on the Management of Patients with Unstable Angina) [erratum published in $7 \mathrm{Am}$ Coll Cardiol 2001;38:294-5]. $7 \mathrm{Am}$ Coll Cardiol 2000;36:970-1062.

4. Fitchett D, Goodman S, Langer A. New advances in the management of acute coronary syndromes: 1 . Matching treatment to risk. CMAf 2001;164(9):1309-16.

Competing interests: None declared.

DOI:10.1503/cmaj.1041116

\section{[Four of the authors respond:]}

$T$ he study by Steve Goodacre and his colleagues ${ }^{1}$ addresses the issues of safe and efficient discharge decisions for patients with chest pain. Their data suggest that a chest pain unit is costeffective compared with routine care in a British emergency department. However, the population in that study appears very different from that of most US chest pain units, especially in terms of the high admission rate after evaluation in the chest pain unit, and it would be difficult to compare the patient population in our study ${ }^{2}$ with the population in the British study. ${ }^{1}$ The Canadian health care system is substantially different from both the US and the UK models, and we maintain that a combination of sensible and safe early discharge combined with an efficient ruleout protocol would be more efficient than mandating that a large number of 